\title{
A Meteorological Parameter for Radioclimatological Purposes
}

\author{
Pierre Misme, ${ }^{1,2}$
}

(Received October 17, 1963; revised February 19, 1964)

\begin{abstract}
The author begins with a study of the index of refraction and shows that theoretically this index varies exponentially with altitude above a certain level. Making use of an example chosen at Hilo, Hawaii, he shows that the curve of the index as a function of altitude shows a point of inflection, and this point of inflection is proposed as the lower limit of validity of the exponential model.

Then considering the variation of the altitude of the point of inflection along the two different meridians ( $150 \mathrm{~W}$ and $80 \mathrm{~W}$ ), an effect of season and of latitude is put in evidence.
\end{abstract}

\section{Introduction}

The knowledge of refraction, useful in almost all studies of propagation, has given rise to the creation of modes describing the variation of the index of refraction $n$ with altitude $h$. We know moreover that the bending of radio rays takes place mainly in the most dense layers of the atmosphere, namely near the transmitters and receivers for a ground-toground communications path, and near the transmitters only, for ground-air communication such as with radar [Bean, 1958; Brocks, 1963]. We can recall that for an angle of elevation of $0^{\circ}$ :

30 percent of the total bending is reached at an altitude of less than $100 \mathrm{~m}$.

50 percent of the total bending is reached at an altitude of less than $600 \mathrm{~m}$.

For a transhorizon path of $300 \mathrm{~km}$, which is a current industrial realization, it would suffice that the model chosen be exact in the first kilometer above the ground, the precision decreasing rapidly with altitude. For example, the existence of a layer with an important gradient at $3500 \mathrm{~m}$ is of little importance for the study of refraction on ground-to-ground paths. But if the same model is used for an air-toair path at an altitude of $3000 \mathrm{~m}$ this layer can lead to large errors.

We shall start with a theoretical study of the index of refraction for which we will choose the function

$$
N=(n-1) 10^{6}=f(h) .
$$

${ }_{1}^{1}$ Paper written while a Guest Worker, Central Radio Propagation Laboratory, National Bureau of Standards, Boulder, Colo.

2 Present address: Ingenieur de la Meterologie Centre National d'Etudes des Telecommunications, Paris, France.

\section{Theoretical Study of $N(h)$}

We shall characterize here the specific humidity, $s$, measured in grams of water vapor in $1 \mathrm{~kg}$ of moist air. We know that

$$
s \simeq 622 \frac{e}{P}
$$

where:

$e=$ the vapor pressure of water in $\mathrm{mb}$

$P=$ atmospheric pressure in $\mathrm{mb}$.

If, moreover, $T=$ the absolute temperature in degrees Kelvin, we can write

$$
(n-1) 10^{6}=N=77.6 \frac{P}{T}\left(1+7.7 \frac{s}{T}\right) .
$$

The quantity $P / T$ is proportional to the density of air and is well represented as a function of the altitude up to at least $100 \mathrm{~km}$ by an exponential law of the form

$$
k_{1} e^{-k_{2} h} \text {, }
$$

where $k_{1}$ and $k_{2}$ are positive constants depending on the place and the season. This suggests that we look for an exponential form as a model for $N(h)$. We note that if

$$
7.7 \frac{s}{T}<<1 \text { or } s<<\frac{T}{7.7} \simeq 40 \frac{\mathrm{g}}{\mathrm{kg}},
$$

the exponential term is predominant.

It is easy to show that $s$ tends toward zero as the altitude increases. Hence, there always exists an 
altitude $H$ above which the inequality (3) is true. It follows that the exponential model is always valid above $H$ [Misme, 1960]. At altitudes lower than $H$, we may speak of a "perturbed low layer." In order to determine the thickness of this layer, three methods have so far been successively used.

(a) In (3) we can arbitrarily choose a value $s_{\max }$ such that

$$
s \leq s_{\max }<<40 \mathrm{~g} / \mathrm{kg} .
$$

Noting that $s_{\max }$ is a function of $T$, hence of the altitude, we thus have an evaluation of $H$ through a knowledge of the ground temperature [Misme, 1960].

(b) We may write (1) in the form of two terms, one of which does not contain $s$. We shall call it the "dry term," and the other the "wet term." Assuming that the wet term may also be represented by an exponential law, we calculate the different characteristics of this biexponential model [Bean, 1961].

(c) $H$ may be determined graphically knowing $N(h)$. After having calculated the coefficients $k_{1}$ and $k_{2}$ at high elevations (for example 8 and $20 \mathrm{~km}$ ), we look for the level below which $N(h)$ deviates from an exponential. It is then easy to operate on the derivative $\frac{d N}{d h}$ and practically it is appropriate to fix a value of the deviation between the theoretical exponential curve and the actual curve [Aldunate and Rutland, 1962].

\section{Graphical Method of Estimating Experi- mentally the Thickness of the Perturbed Low Layer}

If in place of working directly with the value $\overline{N(h)}$, we consider $\left(\frac{\overline{d N}}{d h}\right)$, we put in evidence the irregularities of the vertical distribution. In particular the appearance of a point of inflection in $\overline{N(h)}$ is clearly interpreted as a very visible maximum in the curve $\left(\frac{\overline{d N}}{d h}\right)$

Without seeking greater mathematical precision than that which the experimental curves can give, the simple fact that $\left(\frac{\overline{d N}}{d h}\right)$ is monotonic is a good indication of the validity of the experimental model for $N(h)$. The altitude of the point of inflection is an evaluation of the thickness $H$ of the perturbed low layer. By the method of the point of inflection, this evaluation may be an underestimation of $H$, but never an excess.

As an example, figure 1 shows the variation with altitude of the average value $\bar{N}$ for one month and the variation of $\left(\frac{\overline{d N}}{d h}\right)$ for four different months at Hilo (Hawaii).

We note that the graphical determination of $H$ is not easily made from the curve $N(h)$, whereas it is evident from the curve of $\left(\frac{\overline{d N}}{d h}\right)$. Actually the simple reading of numbers $\left(\frac{\overline{d N}}{d h}\right)$ is sufficient to determine $H$. However, to take account of errors in measurement we disregard amplitudes less than one $N$ unit per kilometer.

On figure 2 we have entered the values of $H$ as a function of the different months of the year. We note here a rather slight annual variation which we have roughly estimated by a dashed line.

\section{Estimation of the Thickness of the Per- turbed Low Layer Along a Meridian}

On figure 3 we have shown the value of $H$ for summer (August) and for winter (February) for stations situated approximately along the $150 \mathrm{~W}$ meridian in the northern hemisphere. We should observe that for Barrow (Alaska) the maximum of the curve $\left(\frac{d N}{d h}\right)$ is small $(1.4 \mathrm{~N} / \mathrm{km})$ and not very significant from the statistical point of view.

It is probable that this is due to a ground-sea transition. A dashed line joining the different points has been traced in order to show a rough estimate of the variation of $H$ along the meridian for the two months of the year considered. In spite of the sparsity of information, the effect of latitude and season is essentially determined. It must be noted that the maximum value of $H$ is at few degrees North of the geographic equator which corresponds to the estimated position of the thermal equator.

In figure 4 a similar study has been made for the meridian $80 \mathrm{~W}$. It is interesting to note that:

(a) For northern latitudes above $30^{\circ}$ and for Antofagasta (Chile) in the southern hemisphere where the seasons are reversed, the effect of seasons and of latitude is confirmed.

(b) For summer the perturbed low layer has a thickness of zero at Balboa. We have attempted to generalize this work by studying the stations situated along the equator, but the lack of data gives little value to this study. We can simply state that it is certain that the exponential profile is systematically valid above about $3000 \mathrm{~m}$, and that in certain special cases for certain months this model may be used down to zero altitude.

Conclusions: From this very brief study we can draw several conclusions as follows:

(a) There is always an altitude above which the variation of $N$ with altitude, $N(h)$, can be represented by an exponential model both theoretically and experimentally.

(b) An evaluation below this altitude shows that $H$ for a single exponential model is always zero in the polar regions and increases in the direction of the thermal equator to a value of the order of $3 \mathrm{~km}$.

(c) Lacking information on $\mathrm{N}$ as a function of altitude, one can roughly evaluate $\mathrm{H}$ by the intermediary of an estimated gradient $\left(\frac{d s}{d h}\right)$ or $\left(\frac{d T}{d h}\right)$ in order to satisfy the inequality (3). 


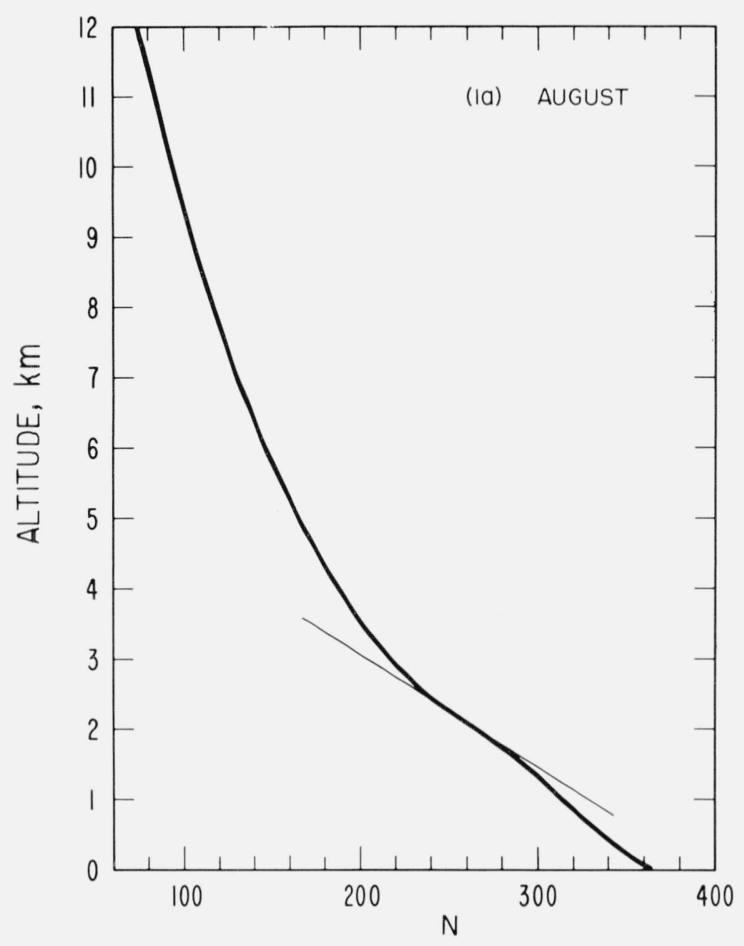

\section{RADIOCLIMATOLOGY OF HILO (HAWAII)}

(Ia) VARIATION OF N AS A FUNCTION OF ALTITUDE FOR THE MONTH OF AUGUST.

THE EXISTENCE OF A POINT OF INFLECTION IS DIFFICULT TO DETERMINE WITH PRECISION.

(Ib) $\left(\frac{\overline{d N}}{d h}\right)$ vs. ALTITUDE, FEBRUARY

(IC) " " " MAY

(Id) " " " AUGUST

(le) " " " NOVEMBER

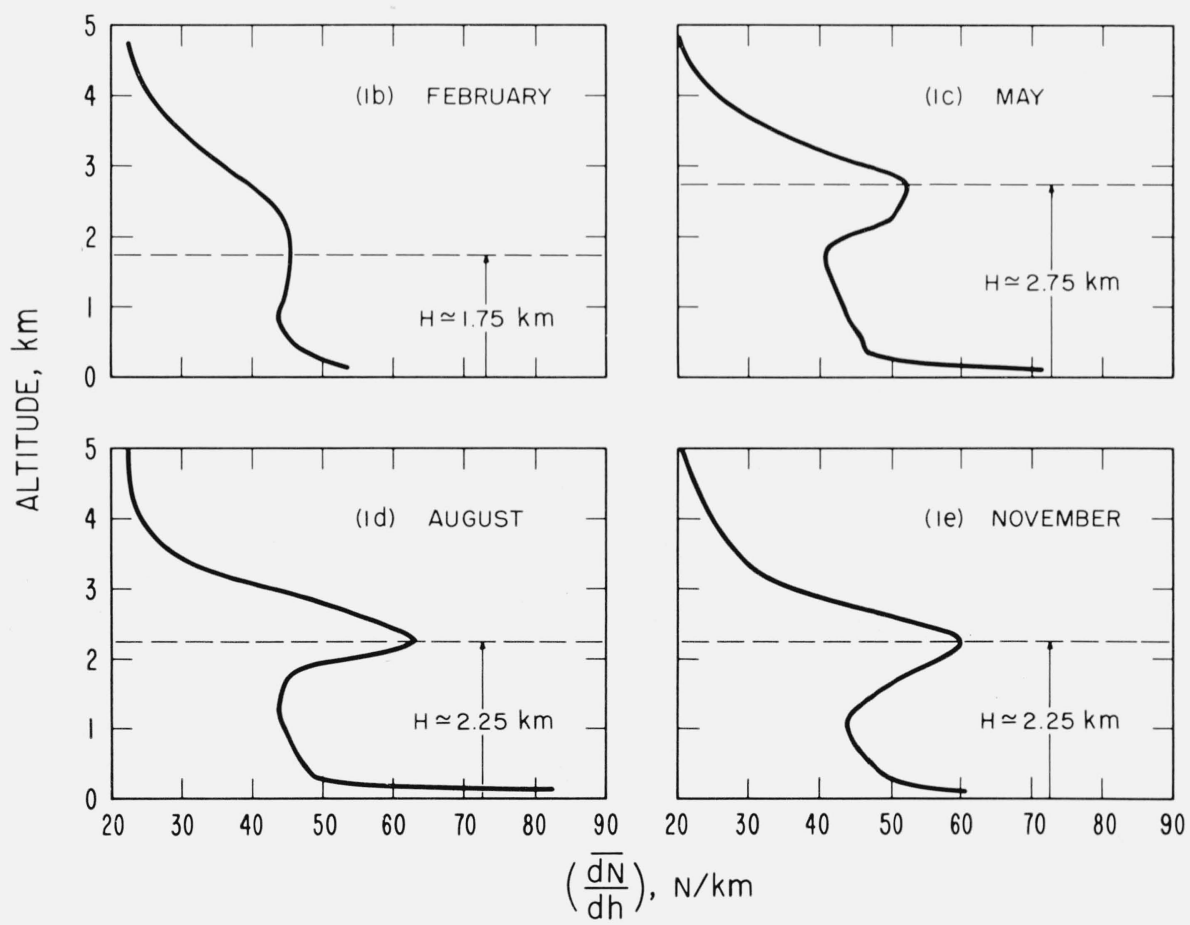

Figure 1. Radioclimatology of Hilo (Hawaii).

1 a. Variation of $N$ as a function of altitude for the month of August. Note the existence of a point of inflection from which it is difficult to estimate the altitude. $1 \mathrm{~b}$. Variation of $d \mathrm{~N} / d h$ as a function of the altitude for February.

$1 \mathrm{c}$. Variation of $d N / d h$ as a function of the altitude for May.

1 d. Variation of $d N / d h$ as a function of the altitude for August.

$1 \mathrm{e}$. Variation of $d N / d h$ as a function of the altitude for November. 


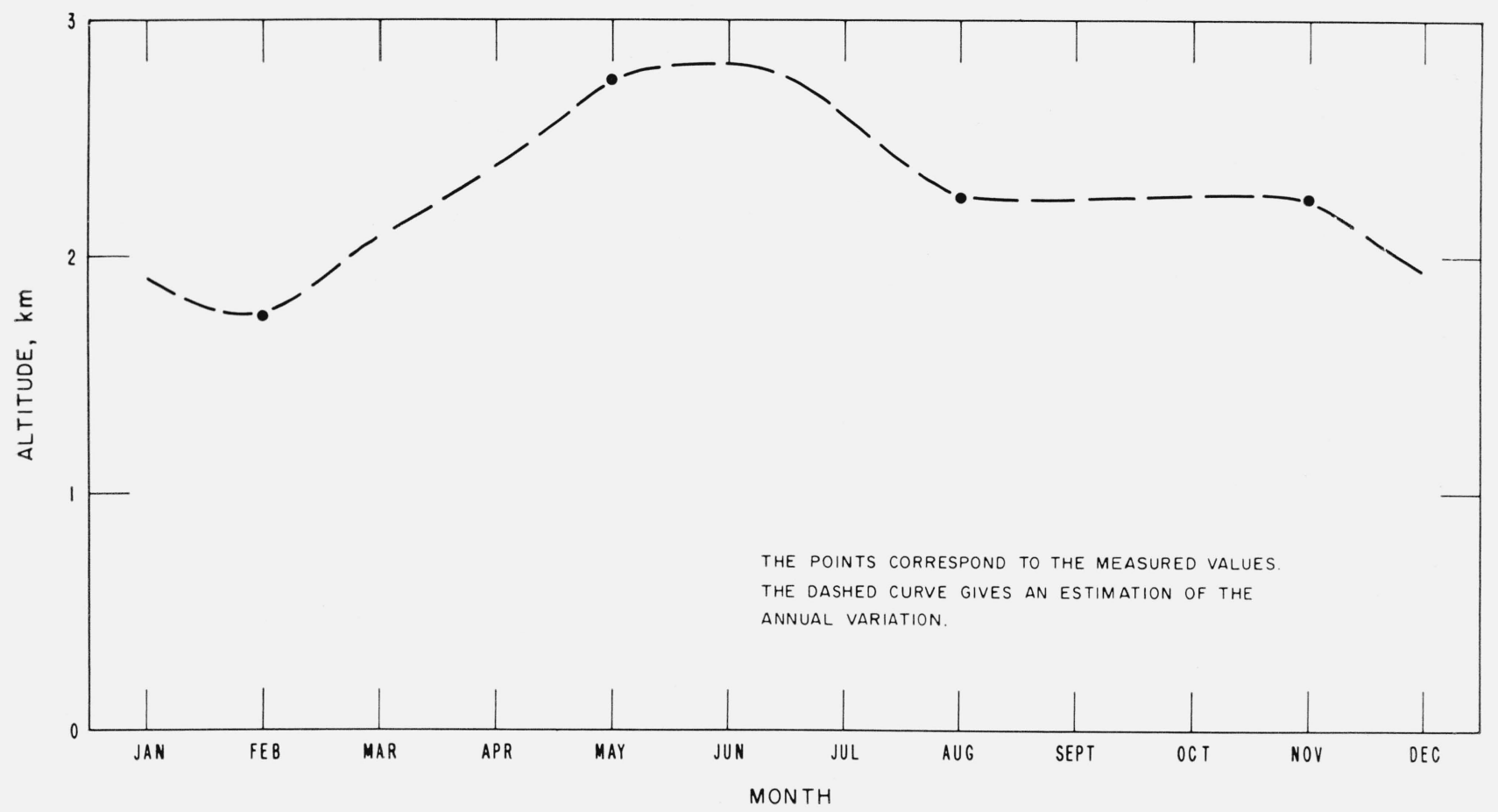

FIgURE 2. Estimation of the annual variation of the altitude of slight inflection of $\mathrm{N}(\mathrm{h})$ at Hilo.

The points correspond to measured values. The discontinuous line gives a rough estimation of the annual variation.

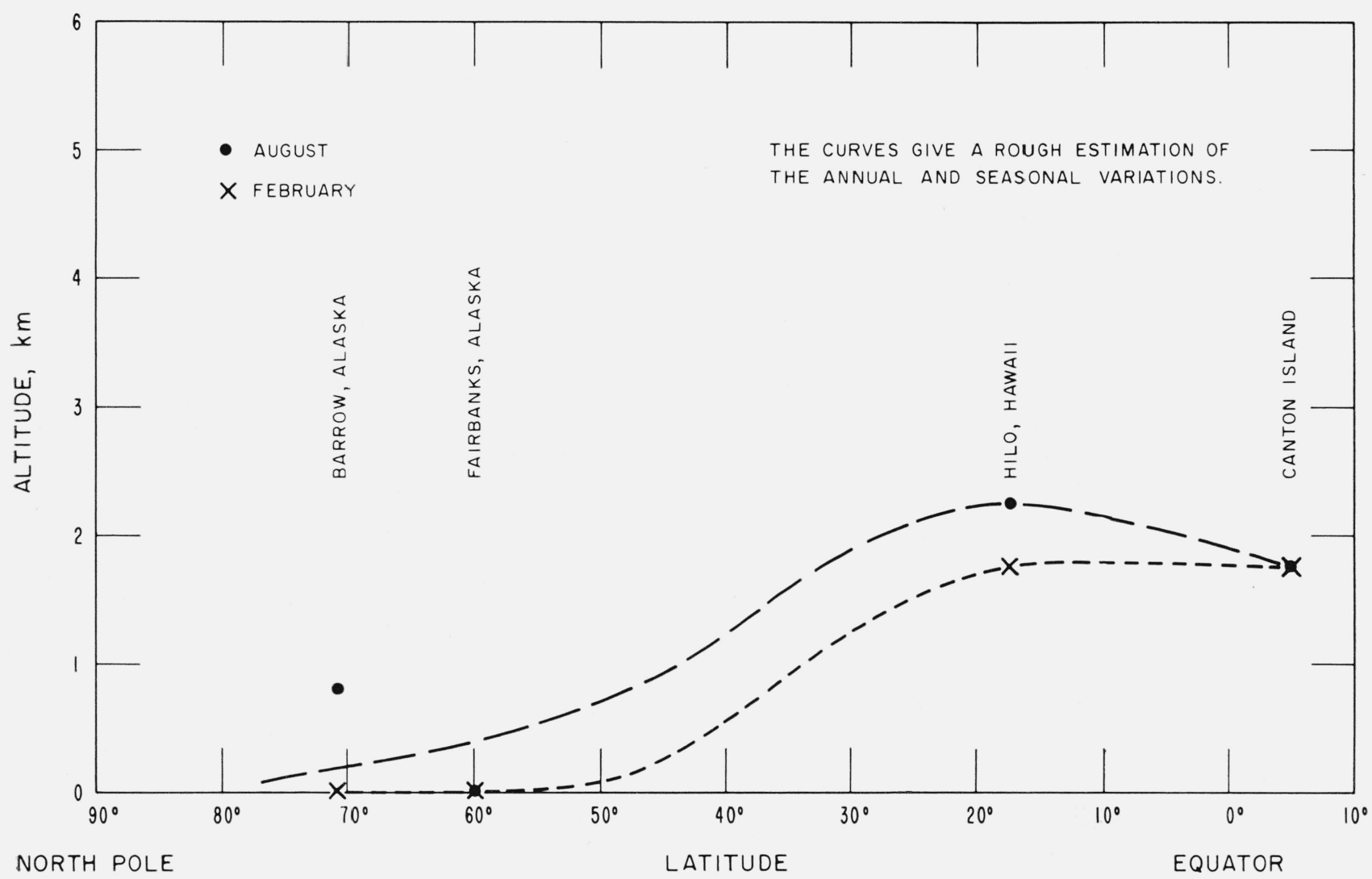

FIGURE 3. Estimation of the meridianal variation of the altitude of slight inflection of $\mathrm{N}(\mathrm{h})$ for the $150 \mathrm{~W}$ meridian. The discontinuous line gives a rough estimate of the annual and seasonal variation. 


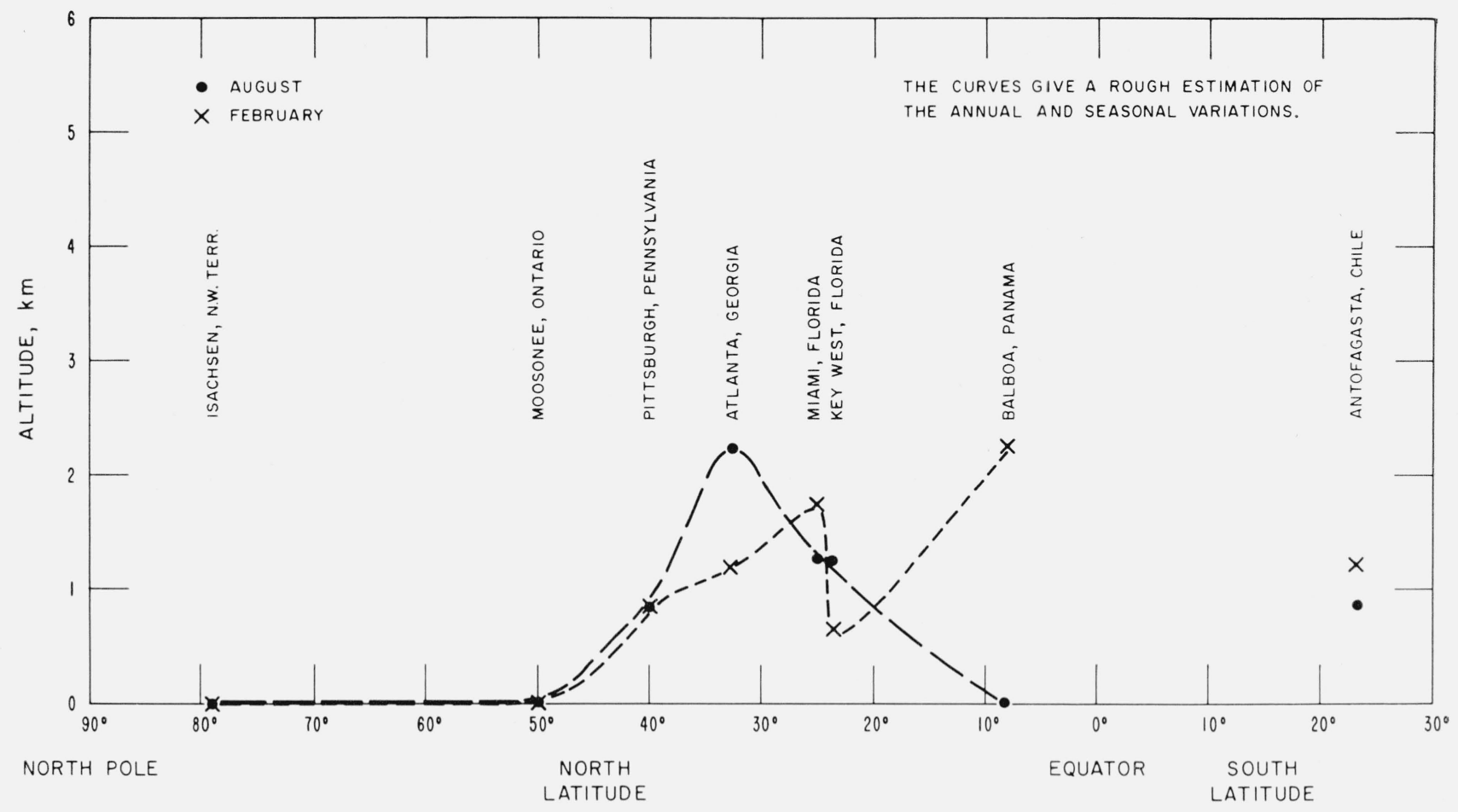

Figure 4. Estimation of the meridianal variation of altitude of the point of inflection of $\mathrm{N}(\mathrm{h})$ for the $80 \mathrm{~W}$ meridian.

The points of the crosses correspond to measured values for two different months. The discontinuous lines give a rough estimation of the annual and seasonal variation. The names of stations used have been given in code.

(d) Bean [1961] has investigated a biexponential model for the perturbed low layer and without entering into the details of verification, it can be stated that the biexponential model is a significant advance over the single exponential model.

In addition it would appear that from the data examined in the present study

above the height $H$ the single exponential model is valid, from ground level to somewhat below the height $H$ the

biexponential model is in essential agreement with the observed $N$ structure,

the $N$ structure near the height $H$ is not well explained by any existing model.

The author thanks the National Bureau of Standards which gave him the opportunity to do this work and especially Messrs. J. W. Herbstreit and B. R. Bean.

\section{References}

Aldunate and Rutland (1962), Report No. 22, Inst. de Geof. y sism., Univ. Chile.

Bean, B. (1958), Electromagnetic Wave Propagation (Academic Press, New York and London).

Bean, B. (1961), Concerning the Bi-exponential nature of the tropospheric radio refractive index, Beiträre, Physik Atmos.

34, $1-2$.

Brocks, K. (1963), Refraction and Ducting, Report of Proceedings, XIIIth General Assembly, International Union of Geodesy and Geophysics, Berkeley, Published by the International Assoc. of Meteorology and Atmospheric Physics, Toronto, Canada.

Misme, P. (Jan. 1960), Influences Radioclimatologiques Sur Les Liaisons Trans-Horizon, L'Onde Electrique 40, No. 394, $116-123$.

(Paper 68D7-383) 